\title{
Limits on Estimating the Width of Thin Tubular Structures in 3D Images
}

\author{
Stefan Wörz and Karl Rohr \\ University of Heidelberg, IPMB, and DKFZ Heidelberg, \\ Dept. Bioinformatics and Functional Genomics, Biomedical Computer Vision Group \\ Im Neuenheimer Feld 364, 69120 Heidelberg, Germany \\ s.woerz@dkfz.de
}

\begin{abstract}
This work studies limits on estimating the width of thin tubular structures in 3D images. Based on nonlinear estimation theory we analyze the minimal stochastic error of estimating the width. Given a 3D analytic model of the image intensities of tubular structures, we derive a closed-form expression for the Cramér-Rao bound of the width estimate under image noise. We use the derived lower bound as a benchmark and compare it with three previously proposed accuracy limits for vessel width estimation. Moreover, by experimental investigations we demonstrate that the derived lower bound can be achieved by fitting a $3 \mathrm{D}$ parametric intensity model directly to the image data.
\end{abstract}

\section{Introduction}

Segmentation and quantification of 3D tubular structures from 3D medical image data is crucial for diagnosis, treatment, and surgical planning. In particular, the accurate quantification of vessels is indispensable. For example, an abnormal narrowing of arteries (stenosis) is one of the main reasons for heart and vascular diseases as the essential blood flow is hindered. For instance, the blocking of a coronary artery can lead to a heart attack. In clinical practice, images of the human vascular system are acquired using different imaging modalities, for example, 3D magnetic resonance angiography (MRA) or computed tomography angiography (CTA). An essential task is the accurate estimation of the width (diameter) of vessels, for example, to identify and quantify stenoses, in particular for thin vessels such as coronary arteries. For a recent review on vessel segmentation techniques we refer to [1].

Concerning thin vessels in 3D images, limits on the accuracy of estimating the vessel width have been studied by different groups using different approaches (e.g., [234], see below for details). However, the results of these studies have not yet been compared with each other. For a quantitative comparison of the proposed limits it would be preferable to have a benchmark. Analytic benchmarks for performance evaluation have been introduced for a different task in medical image analysis, namely, the localization of 3D landmarks in [5].

In this contribution, based on nonlinear estimation theory, we have analyzed the minimal stochastic error of estimating the width of $3 \mathrm{D}$ tubular structures. 
Given a 3D analytic model of the image intensities of a tubular structure as well as white Gaussian image noise, we have derived a closed-form expression for the Cramér-Rao bound (CRB) of the vessel width, which defines the minimal uncertainty of quantifying the width. In this work, we use a cylinder model of 3D tubular structures, which is much more complex compared to the Gaussian landmark models in [5]. In addition, we consider the width of this tubular structure instead of the position of landmarks. We employ the derived CRB as a benchmark and compare it with previously proposed accuracy limits of three different approaches for vessel width estimation [234]. Moreover, by experimental investigations we demonstrate that the derived lower bound can be achieved by fitting a 3D parametric intensity model directly to the image data.

For 2D approaches addressing the accuracy of segmenting thin vessels see, for example, Sonka et al. [6]. Work on accuracy limits of thickness measurements of 3D sheet-like structures such as the vertebral cortical shell or the brain cortex has been described in [78], and for work on the analysis of systematic localization errors of curved surfaces see [9]. Note that the focus in these previous works is on the influence of the point spread function and sampling properties whereas the influence of image noise is not considered.

This paper is organized as follows. In the following section, we derive a closedform expression for the CRB of a cylinder model (Sect. 2). We then present experimental results of model fitting and compare them with the theoretical results given by the CRB. In addition, we demonstrate the applicability of model fitting using different 3D medical MR images (Sect. 3). In Sect. 4, we compare the theoretical results with proposed accuracy limits of three different approaches. Finally, we give a conclusion in Sect. 5.

\section{Cramér-Rao Lower Bound for 3D Cylinder Model}

To derive a benchmark for performance evaluation of 3D segmentation approaches for tubular structures, we use a 3D analytic model that represents the image intensities of tubular structures. The model we employ is an ideal sharp 3D cylinder convolved with a 3D Gaussian, which is well-suited to model tubular structures of different widths (e.g., 34). This 3D cylinder model comprises parameters for the width of the tubular structure (radius $R$ ), the image blur $\sigma$, as well as the image contrast $a$ between the intensity levels of the tubular structure and the surrounding tissue. In contrast to a 3D Gaussian tubular model this model has two separate parameters for the vessel width and the image blur. A $2 \mathrm{D}$ cross-section of this Gaussian smoothed 3D cylinder is defined as

$$
g_{D i s k}(x, y, R, \sigma)=\operatorname{Disk}(x, y, R) * G_{\sigma}^{2 D}(x, y)
$$

where $*$ denotes convolution, $\operatorname{Disk}(x, y, R)$ is a two-valued function with value 1 if $r \leq R$ and 0 otherwise (for $r=\sqrt{x^{2}+y^{2}}$ ), as well as the 2D Gaussian function $G_{\sigma}^{2 D}(x, y)=G_{\sigma}(x) G_{\sigma}(y)$, where $G_{\sigma}(x)=(\sqrt{2 \pi} \sigma)^{-1} e^{-\frac{x^{2}}{2 \sigma 2}}$. By exploiting the 
symmetries of the disk and the 2D Gaussian function as well as the separability of the $2 \mathrm{D}$ convolution, we can rewrite (11) as

$$
\begin{aligned}
g_{\text {Disk }}(x, y, R, \sigma)= & 2 \int_{-R}^{R} G_{\sigma}(r-\eta) \Phi_{\sigma}\left(\sqrt{R^{2}-\eta^{2}}\right) d \eta \\
& -\left(\Phi_{\sigma}(r+R)-\Phi_{\sigma}(r-R)\right)
\end{aligned}
$$

using the Gaussian error function $\Phi(x)=\int_{-\infty}^{x} G_{1}(\xi) d \xi$ and $\Phi_{\sigma}(x)=\Phi(x / \sigma)$. Extending the 2D disk in $z$-direction as well as including the image contrast $a$ yields the $3 \mathrm{D}$ cylinder model

$$
g_{M, C y l i n d e r}(\mathbf{x}, R, \sigma, a)=a g_{D i s k}(x, y, R, \sigma),
$$

where $\mathbf{x}=(x, y, z)^{T}$. To determine a lower bound for estimating the radius of the tubular structure, we utilize the Fisher information matrix $\mathbf{F}$. In the case of $3 \mathrm{D}$ images, the Fisher information matrix is given by

$$
\mathbf{F}=\frac{\operatorname{vox}^{-3}}{\sigma_{n}^{2}} \int_{-w}^{w} \int_{-w}^{w} \int_{-w}^{w} \frac{\partial g_{M}(\mathbf{x}, \mathbf{p})}{\partial \mathbf{p}}\left(\frac{\partial g_{M}(\mathbf{x}, \mathbf{p})}{\partial \mathbf{p}}\right)^{T} d \mathbf{x}
$$

where $\sigma_{n}$ denotes the standard deviation of the additive white Gaussian image noise, $\mathbf{p}$ are the considered model parameters, $w$ is the half-width of the cubic region-of-interest (ROI), and vox denotes the spatial unit in $3 \mathrm{D}$ (i.e. one voxel is a cube with a size of one vox in each dimension). Here we are interested in estimating the radius $R$ of the tubular structure, assuming that the values of the remaining parameters are known, i.e. $\mathbf{p}=(R)$. The matrix $\mathbf{F}$ consists of one element and the Cramér-Rao lower bound (CRB) of the uncertainty is given by (e.g., [10])

$$
\sigma_{\hat{R}}^{2} \geq \sigma_{C R B, \hat{R}}^{2}=\mathbf{F}^{-1} .
$$

The bound determines the minimal possible uncertainty of the estimated parameter $R$ for a given level of image noise. For calculating the CRB in (5), the first order partial derivative of the cylinder model w.r.t. the radius $R$ is required. Fortunately, whereas a closed-form solution of the Gaussian smoothed cylinder model in (3) is not known, a closed-form expression of the required partial derivative can be derived analytically:

$$
\frac{\partial g_{M, C y l i n d e r}(\mathbf{x}, R, \sigma, a)}{\partial R}=a \frac{\sqrt{2 \pi} R}{\sigma} G_{\sigma}\left(\sqrt{r^{2}+R^{2}}\right) I_{0}\left(\frac{r R}{\sigma^{2}}\right),
$$

with $I_{0}$ being the modified Bessel function of the first kind of order 0 (e.g., 11]).

Here, we consider cylindrical ROIs of half-width (radius) $w$ within the $x y$ plane and half-width $w_{z}$ in $z$-direction (along the cylinder) around a position on the centerline of the cylinder. A cylindrical ROI as compared to a cubic (or cuboidal) ROI is a more natural choice for tubular structures and also reduces the complexity in the calculation of the involved integrals since cylindrical coordinates can be used. Assuming that the half-width $w$ of the ROI (within the 


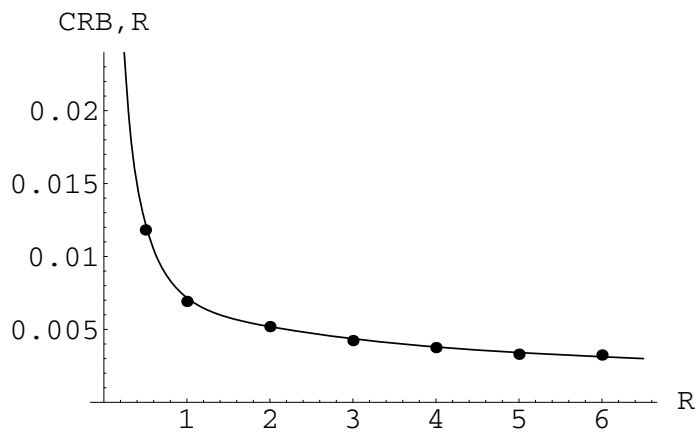

Fig. 1. Theoretical and experimental precision for estimating the radius $R$ as a function of the radius $R$, using $a=100 \mathrm{gr}, \sigma=1 \mathrm{vox}, \sigma_{n}=5 \mathrm{gr}$, and $w_{z}=12$ vox.

$x y$-plane) is much larger than the radius $R$ and the standard deviation $\sigma$, the closed-form expression of the CRB in (5) using (6) can be stated as

$$
\sigma_{C R B, \hat{R}}^{2}=\frac{\sigma^{2} \sigma_{n}^{2} e^{\frac{R^{2}}{2 \sigma^{2}}}}{2 \pi a^{2} R^{2} w_{z} I_{0}\left(\frac{R^{2}}{2 \sigma^{2}}\right)} \operatorname{vox}^{3}
$$

It can be seen that the precision increases (i.e. the bound decreases) with decreasing image noise $\sigma_{n}$ as well as increasing image contrast $a$ and size $w_{z}$ of the $3 \mathrm{D}$ ROI along the cylinder, and depends in a more complicated way on the radius $R$ and the image blur $\sigma$ (compared to $\sigma_{n}, a$, and $w_{z}$ ). The limit of $\sigma_{C R B, \hat{R}}^{2}$ in (7) for $R \rightarrow 0$ is $\infty$, and the limit for $R \rightarrow \infty$ is 0 . The curve in Fig. 1 visualizes the $\mathrm{CRB}$ in terms of the standard deviation (square root of the variance) $\sigma_{C R B, \hat{R}}$ as a function of the radius $R$. It can be seen that the bound is monotonically decreasing as a function of the radius $R$.

To give an impression of the achievable accuracy, we state numerical examples of the $\mathrm{CRB}$ of the radius for thin tubular structures. For example, using $a=$ $100 \mathrm{gr}, \sigma=1$ vox, $\sigma_{n}=5 \mathrm{gr}$, and $w_{z}=12$ vox, for radii $R$ of $0.5,1,2$, and $3 \mathrm{vox}$ the minimal uncertainties $\sigma_{C R B, \hat{R}}$ compute to $0.012,0.007,0.005$, and 0.004 vox, respectively ( $g r$ denotes the unit of the intensities in grey levels). Thus, for thin tubular structures the precision is well in the subvoxel range. However, for very thin tubular structures (e.g., a width of 1 vox) in combination with extremely poor imaging conditions (i.e. a very poor signal-to-noise ratio), the uncertainty of the radius can excess the radius of the tubular structure itself. For example, for an extremely low image contrast of $a=5 \mathrm{gr}$, a radius of $R=0.5$ vox, and a small size of the ROI along the cylinder of $w_{z}=5$ vox, the CRB computes to 0.76 vox, which is about $50 \%$ larger in comparison to the radius.

Note that the derived CRB in (7) does not impose a general limit for small radii of tubular structures (i.e. the equation is valid for $R \rightarrow 0$ ). However, a limit for small radii can be determined based on the desired maximal uncertainty of the radius (e.g., $5 \%$ or 0.1 vox). For example, for a maximal uncertainty of $5 \%$, the 

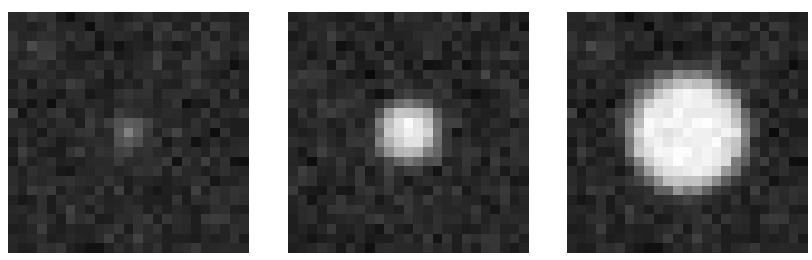

Fig. 2. 2D axial sections of 3D synthetic cylinders of radii $R=1$ (left), $R=3$ (middle), and $R=6$ (right), using $a=100 \mathrm{gr}, \sigma=1 \mathrm{vox}, \sigma_{n}=5 \mathrm{gr}$, and $w_{z}=12 \operatorname{vox}$

limit computes to $R=0.34$ vox (using the same parameter settings as above), i.e. the minimal width of $2 R=0.68$ vox is below image resolution.

\section{3D Width Estimation by Model Fitting}

\subsection{D Synthetic Image Data}

We have carried out experiments to analyze how the theoretical bound described above relates to practice. To this end, we have generated 3D images based on the $3 \mathrm{D}$ cylinder model $g_{M, C y l i n d e r}$ in (3) with additive white Gaussian noise for different radii $R=0.5,1,2,3,4,5,6$ vox and using the same parameter settings as above (i.e. $a=100 \mathrm{gr}, \sigma=1$ vox, $\sigma_{n}=5 \mathrm{gr}$ ). Fig. 2 shows $2 \mathrm{D}$ axial sections of 3D synthetic cylinders of radii $R=1$ vox (left), $R=3$ vox (middle), and $R=6$ vox (right). To estimate the radius of $3 \mathrm{D}$ tubular structures we apply a model fitting approach [4] using a cylindrical ROI of size $w=w_{z}=12$ vox. A closed-form solution of the Gaussian smoothed cylinder $g_{M, C y l i n d e r}$ is not known. Whereas in 4. an analytic approximation has been used, we here employ numeric integration to compute the model function. Thus we have the same model for the theoretical analysis and the experiments. In total, for each value of the radius we carried out $N=1000$ experiments (randomly varying the initial model parameters as well as the noise level) and determined the precision $\sigma_{\hat{R}}$ of the radius by the standard deviation of the estimated radii $\hat{R}_{i}$, i.e.

$$
\sigma_{\hat{R}}=\sqrt{\frac{1}{N-1} \sum_{i=1}^{N}\left(\hat{R}_{i}-\bar{R}\right)^{2}},
$$

where $\bar{R}$ denotes the mean.

From the experiments it turned out that the radius estimate is unbiased, i.e. the systematic error is zero. The estimated precision $\sigma_{\hat{R}}$ of the radii are represented by the dots in Fig. 1. The curve indicates the theoretical precision according to the derived $\mathrm{CRB} \sigma_{C R B}, \hat{R}$. It turns out that the agreement between the theoretical and the experimental values is very good, in particular, for thin vessels (e.g., $R=0.5$ vox), i.e. the derived lower bound can indeed be achieved experimentally. The agreement is even more remarkable since the analytic derivation does not consider discretization effects due to sampling and quantization, while in the experiments discrete images have been used. 

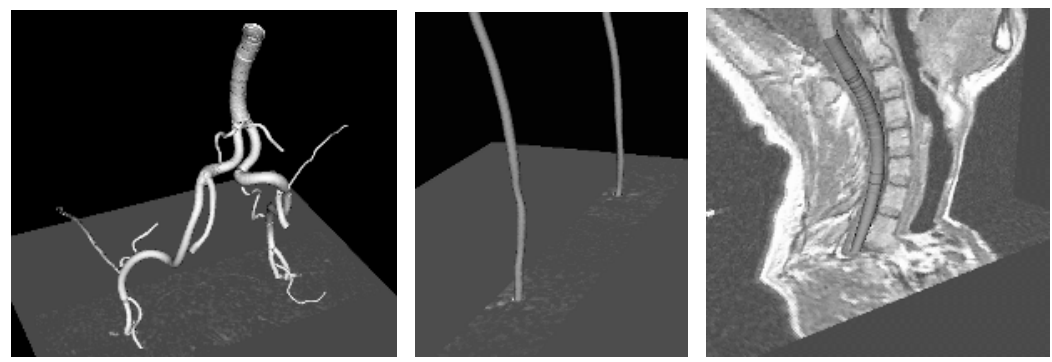

Fig. 3. Segmentation results of applying the 3D cylindrical intensity model to arteries of the pelvis (left), arteries of the thigh (middle), and to the spinal cord (right)

\subsection{D Medical MR Images}

To demonstrate the applicability for real images, we have also applied the model fitting approach to 3D MR image data of the human (see Sect. 4 below for a brief description of the model fitting approach). For reasons of computational complexity we here use an analytic approximation of the Gaussian smoothed cylinder $g_{M, C y l i n d e r}$ as in [4. As a consequence, model fitting is about 80 to 300 times faster (depending on the size of the 3D ROI) in comparison to numerically integrating the model function. As an example, Fig. 3 shows the segmentation results for arteries of the pelvis (left) as well as for thigh arteries (middle) in 3D MRA images. It can be seen that arteries of quite different sizes including relatively thin arteries have been successfully segmented. While above we considered the segmentation of blood vessels, Fig. 3 (right) shows the segmentation result for a different type of tubular structure. Here we successfully segmented the spinal cord in a 3D MR image of the neck.

\section{Comparison of Different Limits}

Having derived an analytic benchmark for 3D vessel width estimation and having shown that the benchmark can be achieved in practice, we here analyze accuracy limits of three different vessel segmentation approaches and compare them with the analytic benchmark. Hoogeveen et al. 2 studied accuracy limits in determining the vessel width from time-of-flight (TOF) and phase-contrast (PC) 3D MRA images. Experiments were based on 3D synthetic TOF and PC MRA images as well as on real images, which were generated by using phantom tubes with known diameters. For measuring the vessel width, the criteria full-width-half-maximum and full-width-at- $10 \%$ were applied for TOF and PC images, respectively. The authors state that for both TOF and PC MRA images a minimal radius of about 1.5 vox is required for accurate estimation of the vessel width (allowing a maximal error of the estimated vessel width of $5 \%$ ). In a second study focusing on model-based estimation of the volume flow rate from PC MRA images, Hoogeveen et al. [12] also provide results for estimating the vessel width. For real PC MRA images of vertebral and basilar arteries, the 
authors state that their approach provides accurate vessel width estimates down to a radius of about 1.5 vox, which is similar to the result in [2].

Sato et al. 3 developed a differential vessel segmentation approach based on a multi-scale line filter. A Hessian-based line filter is applied to different scales of a 3D image and vessels are extracted based on these filter responses. To determine the width of a vessel, the filter responses are compared to filter responses of an ideal vessel model. It turns out that a maximum response of the multi-scale filter, which is required to estimate the vessel width, is inherently not obtainable for thin vessels with a radius below 1.39 vox.

In [4, we developed a model fitting approach for vessel segmentation based on an analytic 3D parametric intensity model. A 3D Gaussian smoothed cylinder is used to model the image intensities of a vessel and the surrounding tissue within a 3D ROI. Since a closed-form solution of a Gaussian smoothed cylinder is not known, we used an accurate approximation which switches between two functions, one based on the Gaussian function and the other based on the Gaussian error function. To segment a vessel we utilize an incremental process based on least-squares model fitting as well as linear Kalman filtering. The cylinder model has been applied to segment vessels in 3D MRA and 3D CTA images of the human. However, we obtain ambiguous estimates of the vessel width for a radius below about 1.72 vox. The reason is that for this value we automatically switch the used approximation in our approach.

To summarize, for all three approaches 234 a limit of $R \approx 1.5$ vox has been stated. Note that, in contrast to Hoogeveen et al. [2], in the approach of Sato et al. [3] as well as in our model fitting approach [4], an accuracy limit is given by the approach itself (note also that in both approaches the limits are stated assuming a standard deviation of the Gaussian image smoothing of 1 vox). In comparison, as discussed above, the derived CRB in (7) of the vessel radius does not impose a general limit for small vessel radii such as in 34. Moreover, above we have shown that the theoretically derived CRB has been achieved experimentally for thin tubular structures, in particular, for a radius of 0.5 vox. Therefore, we conclude that the limit of about $R \approx 1.5$ vox proposed by all three approaches in 2344 is not a fundamental limit.

\section{Conclusion}

We have analyzed limits for estimating the width of thin 3D tubular structures such as vessels. We have derived a closed-form expression for the Cramér-Rao bound of the vessel radius and have compared this bound with three previously proposed limits. Moreover, by experimental investigations we have demonstrated that the derived lower bound can indeed be achieved by model fitting of a $3 \mathrm{D}$ parametric intensity model. It turns out that the previously stated limit of $R \approx$ 1.5 vox is not a fundamental limit. Instead, we have shown that it is possible to correctly estimate vessels with a much smaller radius, e.g., $R=0.5$ vox. Of course, to achieve this result certain assumptions have to be met, e.g., intensity homogeneity, straightness of the cylinder, and adherence to the Gaussian noise 
model. However, all other segmentation approaches also have to make certain assumptions, and only under these assumptions the algorithms yield best results. In future work, we plan to study the performance of more complex analytic models of 3D tubular structures.

\section{Acknowledgment}

The MR images have kindly been provided by Philips Research Laboratories Hamburg, Germany as well as by Dr. med. T. Maier and Dr. C. Lienerth, Gemeinschaftspraxis Radiologie, Frankfurt/Main, Germany.

\section{References}

1. Kirbas, C., Quek, F.: A Review of Vessel Extraction Techniques and Algorithms. ACM Computing Surveys 36 (2004) 81-121

2. Hoogeveen, R., Bakker, C., Viergever, M.: Limits to the Accuracy of Vessel Diameter Measurement in MR Angiography. J. of Magnetic Resonance Imaging 8 (1998) 1228-1235

3. Sato, Y., Yamamoto, S., Tamura, S.: Accurate Quantification of Small-Diameter Tubular Structures in Isotropic CT Volume Data Based on Multiscale Line Filter Responses. In: Proc. MICCAI'04. Volume 3216 of Lecture Notes in Computer Science., St. Malo, France, Springer (2004) 508-515

4. Wörz, S., Rohr, K.: A New 3D Parametric Intensity Model for Accurate Segmentation and Quantification of Human Vessels. In: Proc. MICCAI'04. Volume 3216 of Lecture Notes in Computer Science., St. Malo, France, Springer (2004) 491-499

5. Rohr, K.: Fundamental Limits in 3D Landmark Localization. In: Proc. IPMI'05. Volume 3565 of Lecture Notes in Computer Science., Glenwood Springs, CO/USA, Springer (2005) 286-298

6. Sonka, M., Reddy, G., Winniford, M., Collins, S.: Adaptive Approach to Accurate Analysis of Small-Diameter Vessels in Cineangiograms. IEEE Trans. on Medical Imaging 16 (1997) 87-95

7. Dougherty, G., Newmann, D.: Measurement of thickness and density of thin structures by computed tomography: A simulation study. Medical Physics 26 (1999) $1341-1348$

8. Sato, Y., Tanaka, H., Nishii, T., Nakanishi, K., Sugano, N., Kubota, T., Nakamura, H., Yoshikawa, H., Ochi, T., Tamura, S.: Limits on the Accuracy of 3-D Thickness Measurements in Magnetic Resonance Images - Effects of Voxel Anisotropy. IEEE Trans. on Medical Imaging 22 (2003) 1076-1088

9. Bouma, H., Vilanova, A., van Vliet, L., Gerritsen, F.: Correction for the Dislocation of Curved Surfaces Caused by the PSF in 2D and 3D CT Images. IEEE Trans. on Pattern Analysis and Machine Intelligence 27 (2005) 1501-1507

10. van Trees, H.: Detection, Estimation, and Modulation Theory, Part I. John Wiley and Sons, New York, NY/USA (1968)

11. Abramowitz, M., Stegun, I.: Pocketbook of Mathematical Functions. Verlag Harri Deutsch, Thun und Frankfurt/Main (1984)

12. Hoogeveen, R., Bakker, C., Viergever, M.: MR Phase-Contrast Flow Measurement With Limited Spatial Resolution in Small Vessels: Value of Model-Based Image Analysis. J. of Magnetic Resonance in Medicine 41 (1999) 520-528 